\title{
The SEM/FIB Workbench: Nanorobotics System Inside of Scanning Electron or Focussed Ion Beam Microscopes
}

\author{
V. Klocke, Ivo Burkart, Ralf Kaufmann* \\ * Klocke Nanotechnik, Pascalstr. 17, 52076 Aachen, Germany
}

A good part of the understanding about material functions and process technologies was developed by handling and preparing materials under light microscopes. But material properties and functionalities also depend on structure dimensions that are smaller than the wavelength of light.

At light microscopes it is natural for every expert to use tool sets like tweezers, knives, hooks, probes and several different measurement tools. Without such handling, manipulation and manufacturing tools many present-day products and methods would not exist: No eye surgery, no wristwatch, no in vitro fertilization, just to mention a few. The operators of SEM, FIB or Dual Beam systems generally work without tool sets and call it natural, although the wavelength limit of light is no physical boundary for using such tools. It can be imagined how technology would be pushed when the in-situ SEM/FIB Nanorobotics reach the same degree of usage as tool sets at light microscopes.

The SEM/FIB Workbench was developed step by step within a set of European Research Networks ("RoboMat, RoboSem, NanoHand, Hydromel") and forms now for the first time one in-SEM/FIB Nanorobotics system for in-situ Nanomanipulation, object handling, material preparation \& processing, assembly and manufacturing together with new methods for nano-characterization.

\section{Challenges:}

In general the success of in-SEM/FIB Nanorobotics depends on the combination of several important features in one global system. The main demands for such a system are:

- Nanomanipulators including automation, for the movement of endeffectors or sample handling,

- Plenty of endeffectors for nano- probing, cutting, cleaning, force distance or wear measurements, gripping, sorting or preparation,

- In-situ tip cleaning process, e.g. for continuous nano-manipulation or nano-cutting,

- 3D tool and sample position detection, sample topography and roughness measurements,

- Modular design for fast configuring of nano-analytical or nano-handling processes,

- Precise control of all tool positions including SEM/FIB sample stage in global coordinates,

- SEM picture assisted manipulation by "Live Image Positioning",

- Assistants and automation modules for Nanorobotics and SEM/FIB for secure and easy usage.

The SEM/FIB Workbench enables new interdisciplinary research and development fields, including material \& forensic research, nano-biology \& bionics, pharmacy, pathology, tribology, geology and semiconductor technology.

Several examples will be described during the presentation as review about already realized applications. Some examples thereof are highlighted now in FIG 1: 


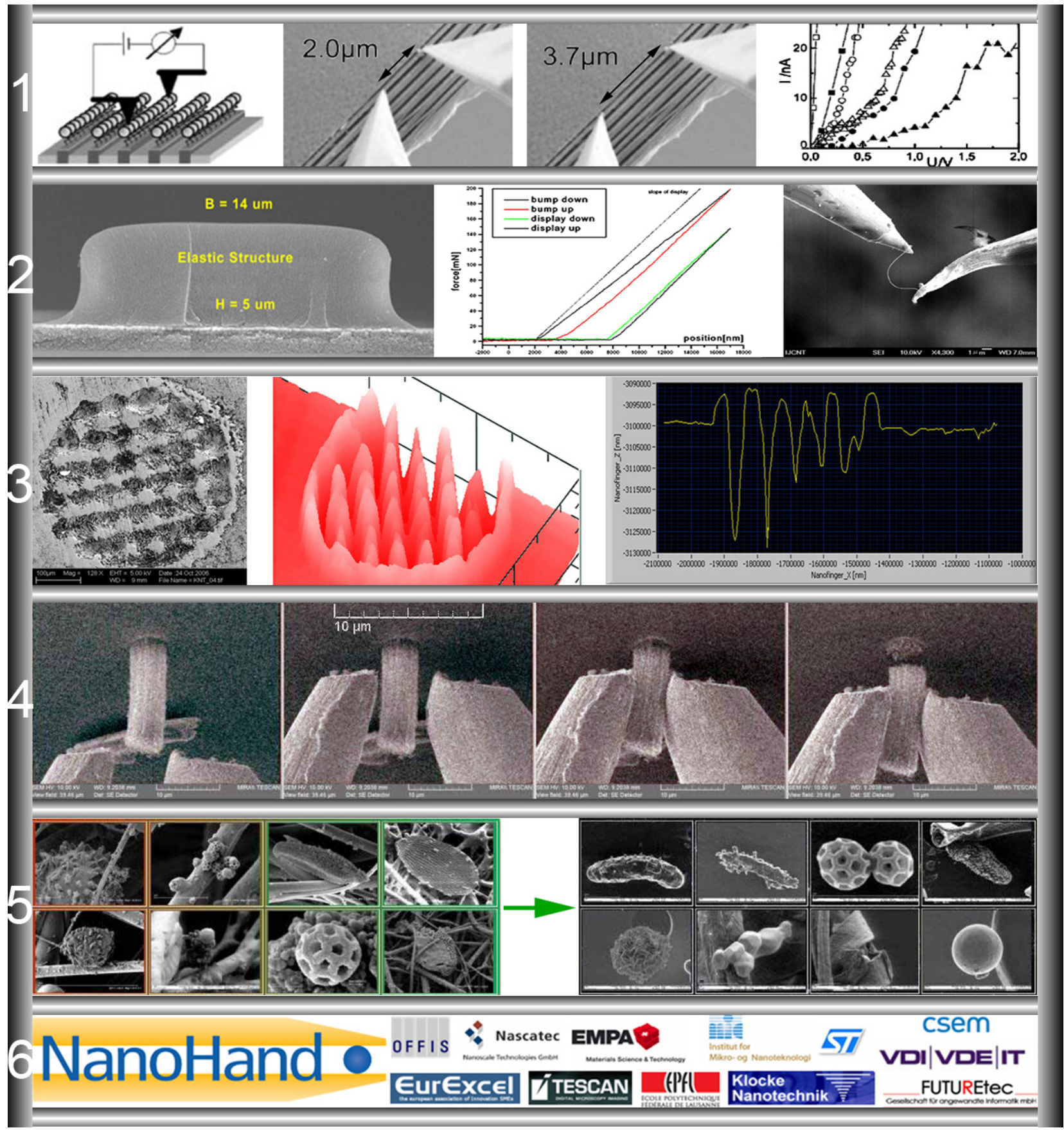

FIG. 1. includes the rows:

1) Nano-Probing of Gold 5 -Clusters arranged in chains,

2) Nano-tribology measurements (friction, reliability, current and heat load, elasticity, ...),

3) SEM image and quantitative 3D-Topography by "Dimensional SEM" module,

4) Gripping of a rigid CNT bundle and separation from the ground,

5) Particle sorting from source area (left) to clean target area (right),

6) Parts of the SEM/FIB workbench were developed in the European Research project "NanoHand", in particular the "NanoFab" module. 